\title{
Avaliação da qualidade de vida geral de agentes comunitários de saúde: a contribuição relativa das variáveis sociodemográficas e dos domínios da qualidade de vida
}

\author{
Evaluation of overall quality of life of community health agents: the relative \\ contribution of sociodemographic variables and domains of quality of life
}

\author{
Ana Cláudia G. C. Kluthcovsky ${ }^{1}$, Angela Maria Magosso Takayanagui ${ }^{2}$, Claudia Benedita dos \\ Santos $^{3}$, Fábio Aragão Kluthcovsky ${ }^{4}$ \\ ${ }^{1}$ Médica. Mestre em Enfermagem em Saúde Pública. Professora, Departamento de Enfermagem, Universidade Estadual do Centro-Oeste, Guarapuava, \\ PR. ${ }^{2}$ Enfermeira. Livre-docente, Doutora em Enfermagem. Professora associada, Departamento de Enfermagem Materno-Infantil e Saúde Pública, \\ Escola de Enfermagem de Ribeirão Preto, Universidade de São Paulo (USP), Ribeirão Preto, SP. ${ }^{3}$ Doutora em Estatística. Professora, Departamento \\ Materno-Infantil e de Saúde Pública, Escola de Enfermagem de Ribeirão Preto, USP. ${ }^{4}$ Médico. Mestre em Enfermagem em Saúde Pública. Professor, \\ Departamento de Enfermagem, Universidade Estadual do Centro-Oeste e Faculdade Guairacá.
}

\section{Resumo}

Objetivo: Avaliar a contribuição relativa de cada domínio da qualidade de vida (físico, psicológico, relações sociais e meio ambiente) e das variáveis sociodemográficas para a qualidade de vida geral de agentes comunitários de saúde de um município do interior do Paraná, Brasil.

Método: Estudo descritivo, de corte transversal e com abordagem quantitativa. O grupo de estudo foi composto por 169 agentes (86,2\% do total), que responderam o World Health Organization Quality of Life Instrument Bref, instrumento genérico para avaliar qualidade de vida, proposto pela Organização Mundial da Saúde. Foram utilizados testes de correlação e regressão linear multivariada. O nível de significância adotado para as análises foi de $5 \%$.

Resultados: Das variáveis sociodemográficas analisadas, nenhuma contribuiu de modo significativo para o domínio geral da qualidade de vida. Para os quatro domínios, o que mais contribuiu para a qualidade de vida geral foi o físico, seguido do psicológico e do meio ambiente, os três explicando $47,9 \%$ da variância. O domínio das relações sociais não contribuiu significativamente para a qualidade de vida geral.

Conclusão: Observou-se que a variância da qualidade de vida geral não foi completamente explicada pelas variáveis sociodemográficas e pelos domínios da qualidade de vida. Assim, maior atenção deve ser dada pelos pesquisadores aos diferentes modos de entender qualidade de vida, em especial valorizando métodos de pesquisa e avaliação interdisciplinar.

Descritores: Agente comunitário de saúde, qualidade de vida, Organização Mundial da Saúde.

\begin{abstract}
Objective: This study aimed at evaluating the relative contribution of each life quality domain (physical, psychological, social relationships and environment) and of sociodemographic variables to overall quality of life of community health agents from a municipality located in the State of Paraná, Brazil.

Methods: A descriptive and cross-sectional study was carried out using a quantitative approach. The sample was composed of 169 agents (86.2\% of the total). The World Health Organization Quality of Life Instrument Bref was used as a generic instrument to evaluate quality of life. Correlation tests and multivariate linear regression were used, considering a significance level of $5 \%$.
\end{abstract}


Results: None of the sociodemographic variables significantly interfered with overall quality of life. Among the four domains, the physical domain contributed the most to overall quality of life, followed by the psychological and environment domains, all of them accounting for $47.9 \%$ of variance. The domain of social relationships did not show significant contribution to overall quality of life. Conclusion: It was observed that variance of overall quality of life was not completely explained by sociodemographic variables neither by quality of life domains. Thus, more attention should be given by researchers to different forms of understanding quality of life, especially research methods and interdisciplinary evaluation.

Keywords: Community health agent, quality of life, World Health Organization.

\section{Introdução}

A expansão e a qualificação da atenção básica, estruturada através da estratégia do Programa Saúde da Família (PSF), é uma política prioritária para o Ministério da Saúde consolidar o Sistema Único de Saúde. O objetivo principal é a superação do modelo centrado na doença. A utilização do trabalho em equipe é o elemento-chave para a busca permanente de comunicação e troca de experiências e conhecimentos entre os integrantes das equipes e destes com a população de territórios delimitados. Os profissionais de saúde e agentes comunitários de saúde (ACS) assumem responsabilidade pelos respectivos territórios, em uma clara estratégia de formação de vínculoํ.

Os ACS representam o elo entre a equipe profissional e a comunidade ${ }^{2}$. Trabalham com adscrição de famílias, podendo estar ligados a uma unidade básica de saúde pelo Programa de Agentes Comunitários de Saúde (PACS) ou pelo PSF, como membros da equipe multiprofissional ${ }^{1}$. Devem desenvolver atividades de prevenção de doenças e promoção de saúde, por meio de ações educativas individuais e coletivas junto às famílias pelas quais são responsáveis² .

Iniciado em 1994, o PSF apresentou um crescimento expressivo nos últimos anos, representando uma importante demanda de trabalhadores em saúde no mercado de trabalho. Dados de março de 2007 indicavam 219.379 ACS em atividade no país, presentes em comunidades rurais e urbanas. Esse número torna-se ainda mais significativo se for considerado que, à mesma época, 109.588.882 de brasileiros (59\% da população total estimada) era assistida por ACS, e 5.299 municípios brasileiros ( $95,2 \%$ do total) contavam com $\mathrm{ACS}^{3}$.

Resultados do trabalho desse maior número de equipes e profissionais de saúde podem ser observados em estudos sobre o $\mathrm{PSF}^{4-5}$ e sobre o trabalho dos $\mathrm{ACS}^{6-}$ 9, que revelam melhores resultados em indicadores de saúde materno-infantil, do adulto e satisfação do usuário.

Levando em consideração a importância dos ACS como força de trabalho em saúde e a dimensão do respectivo impacto nos indicadores de saúde, é importante entender a dinâmica de trabalho desses profissionais. O papel de "tradutor" do universo científico ao popular, a entrada no contexto e na problemática familiar das pessoas, a freqüente resistência da população à proposta de mudança de hábitos, conflitos e dificuldades de relacionamento com membros da comunidade e dentro da própria equipe de saúde ${ }^{6,8}$ são alguns exemplos de tensões a que os ACS estão cotidianamente submetidos. Também necessitam atenção para discernir quais informações da comunidade, família e indivíduos atendidos devem ser trazidas ao restante da equipe, de modo a gerar benefícios à comunidade, sendo exigida capacidade de enfrentar o dinamismo dos problemas da realidade sanitária ${ }^{10}$.

O Ministério da Saúde enfatiza a necessidade de qualificação da força de trabalho através da educação permanente, como forma de contribuir para a efetivação da Política Nacional de Saúde'. Além de fatores relacionados à sua formação, destaca-se a importância de se conhecer outros aspectos da vida dos ACS, levando em consideração a pessoa do agente, seus dilemas, dificuldades e realizações, fatores estes influenciados ou não pela natureza peculiar do seu trabalho.

Esse aspecto mais subjetivo do profissional não é levado em consideração de forma sistemática. O aumento do número de estudos sobre ACS, especialmente após 2000, revela uma preocupação dos pesquisadores em caracterizar os agentes e suas respectivas funções, avaliar o processo e resultados do trabalho, entre outros temas. Porém, nenhum estudo foi encontrado na literatura sobre avaliação da satisfação ou qualidade de vida do $\mathrm{ACS}^{11}$.

Assim, destaca-se a relevância de se investigar a qualidade de vida do ACS, pois essas informações podem dar sustentação para a implementação de novas estratégias para a melhoria das condições de vida e trabalho dos ACS e subsidiar políticas públicas de saúde, o que pode gerar um impacto positivo na saúde, tanto dos ACS quanto da população por eles assistida.

Qualidade de vida e padrão de vida foram os objetos de estudo, inicialmente, de cientistas sociais, filósofos e políticos ${ }^{12}$, e enfatizavam os aspectos materiais. $\mathrm{O}$ conceito foi ampliado, paulatinamente, englobando o desenvolvimento socioeconômico e humano (aspecto objetivo) e a percepção individual (aspecto subjetivo) das pessoas a respeito de suas vidas, valorizando, assim, a opinião do indivíduo ${ }^{13}$. 
Muitos termos são utilizados na literatura como sinônimos de qualidade de vida, como bem-estar, felicidade, condições de vida e, mais comumente, satisfação com a vida ${ }^{14}$.

Qualidade de vida é definida pela Organização Mundial da Saúde (OMS) como: "a percepção do indivíduo de sua posição na vida no contexto da cultura e sistema de valores nos quais ele vive e em relação aos seus objetivos, expectativas, padrões e preocupações". Essa definição deixa implícita a idéia de que o conceito de qualidade de vida é subjetivo, multidimensional e inclui elementos de avaliação tanto positivos como negativos ${ }^{12,15}$.

Pode-se avaliar a qualidade de vida geral (QVG) utilizando-se medidas genéricas, sem especificar patologias, ou medidas específicas, que apontam, em geral, para situações relacionadas à qualidade de vida dos indivíduos e à experiência de doenças, agravos ou intervenções médicas, referindo-se a doenças crônicas ou a conseqüências crônicas de doenças ou agravos agudos ${ }^{16}$.

Também há uma distinção entre a qualidade de vida global, as dimensões ou domínios da qualidade de vida e os componentes de cada dimensão $0^{17-19}$. Em modelo proposto por Spilker, a qualidade de vida pode ser vista em três níveis, sendo a percepção geral da qualidade de vida o primeiro nível, cada domínio da qualidade de vida o segundo nível e os componentes específicos de cada domínio o terceiro nível do modelo ${ }^{19}$.

Em função do consenso na literatura a respeito da relação entre o primeiro nível (QVG) e o segundo nível (diferentes domínios) do modelo de qualidade de vida de Spilker ${ }^{20-21}$, este estudo teve como objetivo avaliar a existência e a extensão da contribuição das variáveis sociodemográficas e dos domínios da qualidade de vida, incluindo o físico, o psicológico, as relações sociais e o meio ambiente, na QVG dos ACS.

\section{Método}

Trata-se de um estudo descritivo, transversal, de abordagem quantitativa, do qual participaram ACS de um município localizado no interior do estado do Paraná, na Região Sul do Brasil, com economia baseada na indústria, agropecuária e serviços e uma população de aproximadamente 160.000 habitantes, grande parte residente na área urbana ${ }^{22}$.

O município encontrava-se em Gestão Plena de Atenção Básica Ampliada e, até o mês de dezembro de 2004, contava com 33 equipes de PSF e um total de 196 ACS, dos quais 193 faziam parte do PSF, e três, do PACS. Com uma jornada de trabalho diária de 8 horas, a renda mensal média dos ACS era de $\mathrm{R} \$ 367,78$, o que correspondia a 1,4 salários mínimos vigentes na época.

\section{A coleta dos dados}

O estudo foi analisado e aprovado pelo Comitê de Ética em Pesquisa da Escola de Enfermagem de Ribeirão Preto, da Universidade de São Paulo.

Foi realizado um estudo-piloto com $11 \mathrm{ACS}$, todos do mesmo município onde foi realizada a presente pesquisa. Esses ACS foram esclarecidos sobre a pesquisa, sendo que a mesma orientação foi dada aos sujeitos da investigação, quando da coleta definitiva dos dados. No estudo-piloto, não houve dificuldade para o entendimento das questões do questionário, e os dados coletados foram incluídos na pesquisa.

Não participaram do estudo 20 agentes comunitários que estavam afastados por licença ou em período de férias e sete agentes comunitários que se recusaram a participar.

Assim, do total de 196 ACS, o grupo de estudo ficou composto por $169 \mathrm{ACS}$, ou seja, $86,2 \%$ do total de agentes do município.

Antes de iniciar a coleta dos dados, todos os participantes do estudo, inclusive os do estudo-piloto, assinaram o Termo de Consentimento Livre e Esclarecido $^{23}$. Foram considerados tanto os ACS inseridos em uma equipe do PSF como os do PACS. A coleta de dados foi realizada em encontros previamente agendados por um grupo de auxiliares de pesquisa, previamente treinados, de 5 a 12 de novembro de 2004 .

\section{Instrumento para coleta dos dados}

$\mathrm{O}$ instrumento para coleta de dados utilizado foi o questionário genérico sobre qualidade de vida da OMS, o World Health Organization Quality of Life Instrument Bref (WHOQOL-bref) ${ }^{24}$. O questionário foi respondido pelos ACS individualmente, em um único encontro, de forma auto-administrada, utilizando-se como referência para as respostas as últimas 2 semanas.

O WHOQOL-bref é a versão abreviada do WHOQOL-100, ambos desenvolvidos em estudos multicêntricos pelo Grupo de Qualidade de Vida da Divisão de Saúde Mental da OMS. No Brasil, a versão em português do WHOQOL-bref foi desenvolvida no Centro WHOQOL para o Brasil, sendo realizada segundo metodologia preconizada para a versão desse documento ${ }^{24}$.

A primeira parte do questionário é a ficha de informações sobre o respondente, que caracteriza os sujeitos. A segunda é o questionário, composto de 26 questões. As duas primeiras são chamadas overall ou QVG e, quando calculadas em conjunto, geram um escore independente dos domínios. A primeira (Q1) refere-se à qualidade de vida de modo geral, e a segunda (Q2), à satisfação com a própria saúde. As outras 24 questões estão distribuídas em quatro domínios: físico, 
psicológico, relações sociais e meio ambiente ${ }^{24,25}$. As respostas das questões do WHOQOL-bref apresentam escalas do tipo Likert, com cinco níveis cada uma e pontuação que pode variar de 1 a 5 .

Tanto a QVG quanto os domínios são medidos em direção positiva, assim, escores mais altos indicam melhor avaliação da qualidade de vida. Os escores obtidos podem transformar-se em dois tipos de escalas, uma de 4 a 20 e outra de 0 a $100^{26}$.

A escolha desse questionário como instrumento de coleta de dados para este estudo deveu-se ao fato de o WHOQOL-bref ter sido aplicado na versão em português, mostrando características psicométricas satisfatórias ${ }^{24}$, além de ser abrangente e de rápida aplicação. Além disso, há escassez de instrumentos genéricos validados para avaliação da qualidade de vida, especialmente no campo da saúde do trabalhador.

\section{Análise estatística}

Para a análise estatística das variáveis sociodemográficas em relação à QVG e aos domínios, foram utilizados o coeficiente de correlação de Pearson, o teste $t$ de Student e a análise de variância. O coeficiente de correlação de Pearson foi calculado entre os quatro domínios (físico, psicológico, relações sociais e meio ambiente) e a QVG.

Análises de regressão linear múltipla foram construídas para se verificar a contribuição das variáveis sociodemográficas nos domínios da qualidade de vida e da QVG e para a contribuição dos domínios para a QVG. O nível de significância adotado foi de 5\%.

\section{Resultados}

Do total dos 169 (100\%) ACS estudados, 18 $(10,6 \%)$ eram do sexo masculino, e $151(89,3 \%)$, do sexo feminino. A idade média dos ACS foi de 31,1 anos, com desvio padrão de 8,8 , idade mínima de 19,1 anos e idade máxima de 60,1 anos. Quanto ao nível educacional, $61,5 \%$ apresentou o ensino médio completo, seguido de $18,3 \%$ com o ensino médio incompleto. No que se refere ao estado civil, verificou-se que a maior parte dos $\mathrm{ACS}$ era constituída por casados $(42,6 \%)$, seguidos pelos solteiros $(35,5 \%)$ e por aqueles vivendo como casados (13\%) (Tabela 1).

Tabela 1 - Distribuição dos ACS de um município do interior do Paraná, segundo características sociodemográficas, $2004(n=169)$

\begin{tabular}{lccc}
\hline Característica & $\mathbf{N}^{\circ}$ & $\mathbf{\%}$ & Média (desvio padrão) \\
\hline Idade (em anos) & & & $31,1(8,8)$ \\
Sexo & 151 & 89,3 & \\
$\quad$ Feminino & 18 & 10,6 & \\
$\quad$ Masculino & & & \\
Nível educacional & 7 & 4,1 & \\
$\quad$ Ensino fundamental incompleto & 10 & 5,9 & \\
$\quad$ Ensino fundamental completo & 31 & 18,3 \\
$\quad$ Ensino médio incompleto & 104 & 61,5 \\
$\quad$ Ensino médio completo & 15 & 8,9 \\
$\quad$ Ensino superior incompleto & 1 & 0,6 \\
$\quad$ Ensino superior completo & 1 & 0,6 \\
$\quad$ Não informado & & \\
Estado civil & 72 & 42,6 \\
$\quad$ Casado(a) & 60 & 35,5 \\
$\quad$ Solteiro(a) & 22 & 13,0 \\
$\quad$ Vivendo como casado(a) & 10 & 5,9 \\
$\quad$ Separado(a) & 2 & 1,2 \\
$\quad$ Divorciado(a) & 2 & 1,2 \\
$\quad$ Viúvo(a) & 1 & 0,6 \\
$\quad$ Não informado & & \\
\hline
\end{tabular}

ACS = agentes comunitários de saúde. 
As análises estatísticas demonstraram que as variáveis sociodemográficas (idade, sexo, nível educacional e estado civil) não apresentaram diferenças estatisticamente significativas em relação à QVG. Em relação aos domínios, observou-se apenas diferença significativa entre sexos $(p=0,022)$ para o domínio físico, com sexo feminino apresentando valor médio de avaliação $(75,0 \pm 13,0)$ superior ao sexo masculino $(67,5 \pm 13,1)$.

Quanto à autopercepção da saúde pelos ACS, observou-se que $60,9 \%$ avaliaram como "boa", seguidos de $19,5 \%$ que avaliaram como "muito boa". Contudo, 15,4\% avaliaram de maneira intermediária, e 3,6\%, de maneira desfavorável (fraca ou muito ruim). Apesar desses resultados, 74 (43,8\%) ACS relataram ter algum problema de saúde no momento da coleta de dados. Dentre esses 74 ACS, o problema de saúde mais relatado foi "pressão alta" (21\%), seguido de "problema crônico de pé" (8\%) e "depressão" (6\%).

Observa-se, pela Tabela 2, que, entre os domínios, o das relações sociais apresentou o maior escore médio, seguido pelos domínios físico e psicológico. O domínio que apresentou o menor escore médio foi o do meio ambiente.

Para as análises dos quatro domínios e da QVG, foi realizada a correlação de Pearson. Pela Tabela 3, observa-se que todos os quatro domínios se correlacionaram positiva e significativamente com a QVG, e todas as correlações apresentaram-se de moderada magnitude.

Tabela 2 - Escore médio, desvio padrão, valores mínimos e máximos da QVG e dos domínios do WHOQOL-bref - ACS de um município do interior do Paraná, 2004 (n = 169)

\begin{tabular}{lcccc}
\hline QVG e domínios & Escore médio & Desvio padrão & Valor mínimo & Valor máximo \\
\hline QVG & 69,6 & 14,5 & 0 & 100 \\
Relações sociais & 75,8 & 14,2 & 16,7 & 100 \\
Físico & 74,2 & 13,2 & 35,7 & 100 \\
Psicológico & 74,0 & 11,4 & 41,7 & 100 \\
Meio ambiente & 54,1 & 12,0 &, 188 & 84,4 \\
\hline
\end{tabular}

ACS = agentes comunitários de saúde; $\mathrm{QVG}=$ qualidade de vida geral; WHOQOL-bref = World Health Organization Quality of Life Instrument Bref.

Tabela 3 - Coeficientes de correlação de Pearson e análise de regressão linear múltipla entre os diferentes domínios em relação à qualidade de vida geral - ACS de um município do interior do Paraná, 2004

\begin{tabular}{lccc}
\hline \multirow{2}{*}{ Domínios } & \multicolumn{2}{c}{ Qualidade de vida geral (variável dependente) } \\
\cline { 2 - 4 } & Correlação & \multicolumn{2}{c}{ Regressão linear } \\
\cline { 2 - 4 } & & $\mathbf{R}^{\mathbf{2}} \mathbf{( \% )}$ & $\boldsymbol{\beta}$ \\
\hline Físico & $0,59^{*}$ & 35,0 & $0,29^{*}$ \\
Psicológico & $0,59^{*}$ & 9,1 & $0,25^{*}$ \\
Meio ambiente & $0,52^{*}$ & 3,8 & $0,20^{*}$ \\
Relações sociais & $0,47^{*}$ & 0,8 & 0,11 \\
$\mathrm{R}^{2}$ total (\%) & & 48,7 & \\
\hline
\end{tabular}

$\mathrm{ACS}=$ agentes comunitários de saúde; $\mathrm{R}^{2}=\mathrm{R}$ square. $* \mathrm{p}<0,01$.

A fim de se verificar a contribuição e o quanto cada domínio da qualidade de vida explicou a QVG (variável dependente), foi realizada a análise de regressão linear múltipla. Como as variáveis sociodemográficas não se mostraram significativas para a QVG, como visto anteriormente, elas não foram incluídas nesse modelo.
Entre os quatro domínios, o domínio que mais explicou a QVG foi o físico, com contribuição de 35\%, seguido do psicológico $(9,1 \%)$ e do meio ambiente $(3,8 \%)$. O domínio das relações sociais teve contribuição de $0,8 \%$, porém esta não foi significativa. Os domínios juntos (físico, psicológico, meio ambiente e relações 
sociais) explicaram 48,7\% da QVG. Já os domínios com contribuição significativa somaram $47,9 \%$.

\section{Discussão}

Vários estudos consideram a influência de variáveis sociodemográficas na avaliação da qualidade de vida, como idade e sexo ${ }^{27}$, além do nível de escolaridade e situação conjugal ${ }^{19-20,28}$.

Os ACS deste estudo apresentaram-se predominantemente constituídos por mulheres e adultos jovens, com idade média de 31,1 anos, e a maior parte dos agentes era constituída por casados.

Outros estudos com ACS demonstraram a predominância de mulheres na profissão ${ }^{29,30}$. Parece haver certa resistência por parte da comunidade ao agente comunitário masculino, em especial pelo constrangimento experimentado pelas famílias em revelar certas particularidades do universo feminino a um agente homem e também pela dificuldade de acesso deste às moradias ${ }^{30}$.

A faixa etária de adultos jovens também foi mais freqüente em estudo sobre $\mathrm{ACS}^{29}$. Acredita-se que os agentes com mais idade tendem a conhecer melhor a comunidade, possuir mais vínculos e amizades, porém podem ter algumas inimizades ou conflitos com outros moradores e, por terem seus próprios conceitos sobre o processo saúde/doença, podem ser mais resistentes a novos conceitos relacionados à promoção da saúde. Ao contrário, os agentes mais jovens não conhecem tão bem a comunidade, podendo ter envolvimento menor; entretanto, poderão não ter inimizades e estar mais abertos às mudanças e às novidades ${ }^{29}$. Além disso, o fato de serem contratados por concurso público pode favorecer a entrada de jovens mais habituados às avaliações por provas da rotina escolar.

A maioria $(61,5 \%)$ apresentou escolaridade de ensino médio completo, seguido de $18,3 \%$ com o ensino médio incompleto, médias superiores quando comparadas ao nível educacional dos ACS da Região Sul do país e do Brasil. O nível educacional mais baixo, ensino fundamental incompleto, foi relatado por $4,1 \%$, sendo que $8,9 \%$ dos ACS estavam cursando o ensino superior, percentual superior à média da Região Sul do país e do Brasil ${ }^{31}$. A maior escolaridade também pode ser uma característica introduzida pelo modo de contratação no contexto estudado.

Sendo um estudo transversal, e mesmo não havendo escores da população em geral ou de outros grupos de ACS para comparações, observou-se que as avaliações realizadas pelos ACS deste estudo indicaram avaliações médias boas no que se refere aos escores médios de QVG e para os domínios, quando comparados aos respectivos valores mínimos e máximos.
Nas análises estatísticas das variáveis sociodemográficas idade, sexo, nível educacional e estado civil, em relação à QVG e domínios, observouse que somente a variável sexo mostrou diferença significativa para o domínio físico, com sexo feminino apresentando valor médio de avaliação $(75,0 \pm 13,0)$ superior ao sexo masculino $(67,5 \pm 13,1)$.

Segundo estudo realizado por Pinheiro et al. ${ }^{32}$, as mulheres utilizam mais regularmente um mesmo serviço de saúde, além de apresentarem outros indicadores de acesso favoráveis em relação aos homens, como o número de consultas médicas, consumo de serviços odontológicos e maior busca por serviços de saúde para realização de exames de rotina e prevenção.

Também é provável que as mulheres ACS, pelo trabalho em saúde, tenham acesso facilitado aos serviços e informações em saúde, evitando o surgimento ou agravamento de doenças.

Sobre a auto-percepção do estado de saúde, deve ser observado que a maioria $(80,4 \%)$ dos ACS considerou seu estado de saúde bom ou muito bom; contudo, 43,8\% do total de ACS relataram ter algum problema de saúde. Essa aparente discrepância entre o significativo número de ACS que referiram problemas de saúde e a maior proporção de auto-avaliações positivas do estado de saúde sugere que os problemas de saúde poderiam não estar interferindo de modo importante em suas atividades diárias.

$\mathrm{Na}$ análise da contribuição de cada domínio para a QVG, o domínio que mais explicou a QVG foi o físico, com contribuição de $35 \%$, seguido do psicológico $(9,1 \%)$ e do meio ambiente $(3,8 \%)$. O domínio das relações sociais não teve contribuição significativa.

Devido à maior influência do domínio físico para a QVG dos ACS, destaca-se a importância da capacidade para o trabalho como fator que pode interferir na qualidade de vida, o que pode estar relacionado com a faixa etária predominante de adultos jovens na realidade pesquisada.

O trabalho, em conjunto com a alimentação, a moradia e a educação, constitui uma necessidade básica humana que deve ser atendida para que se possa edificar uma vida com qualidade ${ }^{33}$. Assim, uma pessoa que não realiza um trabalho satisfatório poderá ter dificuldades e dificilmente terá uma vida com qualidade ou aceitável.

O segundo domínio em termos de influência sobre a QVG dos ACS foi o psicológico. Tanto o esgotamento ${ }^{34}$ como o estresse profissional ${ }^{10}$ têm se tornado cada vez mais sérias ameaças para os profissionais da área da saúde, podendo afetar sua saúde, sua qualidade de vida, o rendimento e eficiência no trabalho, além de causar dificuldades de comunicação e insatisfação, com conseqüentes repercussões na qualidade assistencial. 
Segundo Camelo \& Angerami ${ }^{10}$, em trabalho sobre a avaliação do estresse em profissionais do PSF, a autoestima de um indivíduo pode ser influenciada pela satisfação no trabalho. Assim, uma pessoa que esteja com estresse ocupacional poderá levar suas dificuldades para o seu ambiente familiar ou trazê-las para seu ambiente profissional, o que pode traduzir-se em sentimentos de insegurança.

O domínio do meio ambiente apresentou pequena contribuição na QVG dos ACS. Ainda assim, vale ressaltar a questão da remuneração e vínculo do emprego. Em trabalho de Pedrosa \& Teles ${ }^{6}$, os ACS acharam o salário que recebiam insuficiente diante do trabalho por eles realizado. Além disso, salienta-se a importância de uma política de incentivo salarial, bem como a garantia de direitos trabalhistas, pois um funcionário que recebe um salário digno sente-se valorizado, satisfeito e apresenta, conseqüentemente, maior produtividade e melhor desempenho em sua função.

O domínio das relações sociais não foi significativo na contribuição para a QVG dos ACS. Esse domínio é representado no questionário pelo menor número de questões (três), e essa menor representação no número de questões é um fator que o deixa menos estável do ponto de vista psicométrico ${ }^{24}$.

Apesar de o domínio das relações sociais não ter contribuído significativamente para a QVG, sabe-se que os ACS são profissionais diferenciados no sentido das relações sociais, já que ele reside na própria comunidade onde trabalha, desenvolvendo seu trabalho diretamente com a comunidade. Nesse sentido, o relacionamento dos ACS no ambiente de trabalho tem sido especialmente estudado nos últimos anos, em relação às pessoas da comunidade ou aos outros membros da equipe de saúde ${ }^{4,6,9}$.

Em suma, neste estudo, observou-se que os domínios da qualidade de vida diferiram com respeito às respectivas contribuições para a QVG. Contudo, devem ser consideradas suas limitações, como o desenho transversal, não considerando, portanto, as possíveis variações temporais nos domínios e na QVG e o subseqüente efeito dos domínios na QVG, bem como quais outros fatores de importância poderiam estar influenciando a QVG dos ACS.

Paschoal ${ }^{13}$ enfatiza a importância da avaliação da qualidade de vida percebida pela pessoa, valorizando, assim, a opinião do indivíduo. A qualidade de vida teria um conceito diferente de pessoa para pessoa, tendendo a mudar ao longo da vida. Para Fayers \& Machin $^{18}$, a avaliação global de qualidade de vida permite ao indivíduo definir o conceito de uma maneira significativa para si mesmo, além de poder ser responsiva às diferenças individuais. Para isso, requer que o indivíduo considere todos os aspectos de um fenômeno, ignore aqueles que não são relevantes naquela situação e pondere outros aspectos de acordo com seus respectivos valores e objetivos, de forma a fornecer uma medida de avaliação.

Outro fato que necessita atenção foi a limitada contribuição dos domínios na $\mathrm{QVG}^{20,21}$. Isso poderia ser parcialmente explicado pela utilização de um instrumento abreviado de avaliação, com a QVG composta por duas questões e o domínio relações sociais composto por três questões.

Para medir e operacionalizar qualidade de vida, é geralmente aceita a necessidade de se reconstruir o conceito de seus componentes mensuráveis dentro dos vários processos envolvidos na criação da mesma e representar as medidas agregadas (ou separadas) de qualidade de vida como um índice. Desenvolver a compreensão do conceito de qualidade de vida através desses mecanismos e análise dos respectivos componentes requer inevitavelmente o emprego de pressupostos de avaliação sobre a relação das partes componentes entre si e com a qualidade de vida como um todo ${ }^{17}$.

Arnold et al. ${ }^{20}$ relataram que prejuízos em um ou mais domínios da qualidade de vida não resultam necessariamente em prejuízo da QVG e que a escolha de uma determinada medida de avaliação depende do tipo de informação que se está procurando, como, por exemplo, se a ênfase são os aspectos físicos ou o bemestar geral dos pacientes.

Apesar das dificuldades conceituais, parece claro que qualidade de vida é eminentemente interdisciplinar, sendo necessária a contribuição de diferentes áreas do conhecimento para o aprimoramento metodológico e conceitual $^{35}$.

Este estudo e outros nessa direção podem dar sustentação para a implementação de novas estratégias para a melhoria das condições de vida e trabalho dos ACS, o que, sem dúvida, poderá trazer um impacto nas ações desempenhadas por esses profissionais e, conseqüentemente, na saúde pública do Brasil, além de poder servir de motivação para novas pesquisas, utilizando-se combinação de outros instrumentos para coleta de dados, bem como outras abordagens metodológicas.

\section{Referências}

1. Brasil, Ministério da Saúde. Atenção básica e a saúde da família. Brasília: Ministério da Saúde. Disponível em: http:// dtr2004.saude.gov.br/dab/atencaobasica.php\#acs. Acessado abr 2007.

2. Brasil, Ministério da Saúde. Modalidade de contratação de agentes comunitários de saúde: um pacto tripartite. Brasília(DF): Ministério da Saúde; 2002

3. Brasil, Ministério da Saúde. Agentes comunitários de saúde, equipes de saúde da família e equipes de saúde bucal, em atuação 
- competência março/2007. Brasília: Ministério da Saúde; Disponível em: http://dtr2004.saude.gov.br/dab/documentos/ resumo_por_uf_03_2007.pdf. Acessado abr 2007.

4. Trad LAB, Bastos ACS, Santana EM, Nunes MO. Estudo etnográfico da satisfação do usuário do Programa de Saúde da Família (PSF) na Bahia. Cienc Saúde Coletiva. 2002;7(3):581-9.

5. Moura ERF, Holanda Jr. F, Rodrigues MSP. Avaliação da assistência pré-natal oferecida em uma microrregião de saúde do Ceará, Brasil. Cad Saúde Pública. 2003;19(6):1791-9.

6. Pedrosa JIS, Teles JBM. Consenso e diferenças em equipes do Programa Saúde da Família. Rev Saúde Pública. 2001;35(3):30311.

7. César JA, Cavaleti MA, Holthausen RS, Lima LGS. Mudanças em indicadores de saúde infantil em um município com agentes comunitários: o caso de Itapirapuã Paulista, Vale do Ribeira, São Paulo, Brasil. Cad Saúde Pública. 2002;18(6):1647-54.

8. Nunes MO, Trad LB, Almeida BA, Homem CR, Melo MCIC. O agente comunitário de saúde: construção da identidade desse personagem híbrido e polifônico. Cad Saúde Pública. 2002;18(6):1639-46.

9. Levy FM, Matos PES, Tomita NE. Programa de agentes comunitários de saúde: a percepção de usuários e trabalhadores da saúde. Cad Saúde Pública. 2004;20(1):197-203.

10. Camelo SHH, Angerami ELS. Sintomas de estresse nos trabalhadores atuantes em cinco núcleos de saúde da família. Rev Latinoam Enfermagem. 2004;12(1):14-21.

11. Kluthcovsky ACGC, Takayanagui AMM. Community health agent: a literature review. Rev Lat Am Enfermagem. 2006;14(6):957-63.

12. Fleck MPA, Leal OF, Louzada S, Xavier M, Chachamovich E, Vieira G, et al. Desenvolvimento da versão em português do instrumento de avaliação de qualidade de vida da OMS (WHOQOL100). Rev Bras Psiquiatr. 1999;21(1):19-28.

13. Paschoal SMP. Qualidade de vida no idoso: elaboração de um instrumento que privilegia sua opinião. [dissertação]. São Paulo: Faculdade de Saúde Pública/Universidade de São Paulo; 2001.

14. Meeberg GA. Quality of life: a concept analysis. J Adv Nurs. $1993 ; 18(1): 32-8$.

15. The WHOQOL Group. What Quality of Life? World Health Forum. WHO: Geneva; 1996. p. 354-56.

16. Minayo MCS, Hartz ZMA, Buss PM. Qualidade de vida e saúde: um debate necessário. Ciênc Saúde Coletiva. 2000;5(1):7-18.

17. Rogerson RJ. Environmental and health-related Quality of Life: Conceptual and methodological similarities. Soc Sci Med. 1995;41(10):1373-82.

18. Fayers PM, Machin D. Principles of measurement scales. In: Fayers PM, Machin D. Quality of life: assessment, analysis and interpretation. New York: John Wiley; 2000. p. 28-42.

19. Suurmeijer TP, Reuvekamp MF, Aldenkamp BP. Social functioning, psychological functioning, and Quality of Life in epilepsy. Epilepsia 2001;42(9):1160-8.
20. Arnold R, Ranchor AV, Sanderman R, Kempen GI, Ormel J, Suurmeijer TP. The relative contribution of domains of quality of life to overall quality of life for different chronic diseases. Qual Life Res. 2004;13(5):883-96.

21. Pereira RJ, Cotta RMM, Franceschini SCC, Ribeiro RCL, Sampaio $\mathrm{RF}$, Priore S E et al. Contribuição dos domínios físico, social, psicológico e ambiental para a qualidade de vida global de idosos. Rev Psiquiatr Rio Gd Sul. 2006;28(1):27-38.

22. Instituto Brasileiro de Geografia e Estatística (IBGE). Cidades; Disponível em: http://www.ibge.gov.br/cidadesat/. Acesso mai 2007.

23. Brasil, Ministério da Saúde, Conselho Nacional de Saúde, Comissão Nacional de Ética em Pesquisa (CONEP). Normas para pesquisa envolvendo seres humanos (Res. CNS 196/96 e outras). Brasília (DF): Ministério da Saúde; 2000.

24. Fleck MPA, Louzada S, Xavier M, Chachamovich, Vieira G, Santos $\mathrm{L}$, et al. Aplicação da versão em português do instrumento abreviado de avaliação da qualidade de vida "WHOQOL-bref". Rev Saúde Pública. 2000;34(2):178-83.

25. World Health Organization. WHOQOL: measuring quality of life. Geneva: WHO; 1997 (MAS/MNH/PSF/97.4).

26. World Health Organization. WHOQOL-BREF: introduction, administration, scoring and generic version of the assessment. Geneva: WHO; 1996 (MAS/MNH/PSF/97.4).

27. Nunes MF, Freire MCM. Qualidade de vida de cirurgiões-dentistas que atuam em um serviço público. Rev Saúde Pública. 2006;40(6):1019-26.

28. Sprangers MA, de Regt EB, Andries F, van Agt HM, Bijl RV, de Boer JB, et al. Which chronic conditions are associated with better or poorer quality of life? J Clin Epidemiol. 2000;53(9):895-907.

29. Ferraz L, Aerts DRGC. O cotidiano de trabalho do agente comunitário de saúde no PSF em Porto Alegre. Ciênc Saúde Coletiva. 2005;10(2):347-55.

30. Bezerra AFB, Espírito Santo ACG, Batista Filho M. Concepções e práticas do agente comunitário na atenção à saúde do idoso. Rev Saúde Pública. 2005;39(5):809-15.

31. Conselho Nacional de Secretários Municipais de Saúde. Escolaridade dos ACS por Estado. Informativo CONASEMS. Brasília: CONASEMS; 2004.

32. Pinheiro RS, Viacava F, Travassos C, Brito AS. Gênero, morbidade, acesso e utilização de serviços de saúde no Brasil. Ciênc Saúde Coletiva 2002;7(4):687-707.

33. Nahas MV. A era do estilo de vida. In: Nahas MV. Atividade física, saúde e qualidade de vida: conceitos e sugestões para um estilo de vida ativo. Londrina: Midiograf; 2001. p. 5-22.

34. Caballero Martín M, Bermejo Fernández F, Nieto Gómez R, Caballero Martínez F. Prevalencia y factores asociados al burnout en un área de salud. Aten Primaria. 2001;27(5):313-7.

35. Seidl EMF, Zannon CMLC. Qualidade de vida e saúde: aspectos conceituais e metodológicos. Cad Saude Publica. 2004;20(2):580-8. 